\title{
Deep Search for Optical Counterparts of Gamma-ray Bursters
}

\author{
V.V. Sokolov ${ }^{1}$, A.I. Kopylov ${ }^{1}$, V.G. Kurt ${ }^{2}$, S.V. Zharykov ${ }^{1}$ \\ 1. Special Astrophysical Observatory of Russian Acad. of Sci., Nizhnij Arkhyz, Karachai- \\ Cherkessia 357147, Russia \\ 2 Astro Space Center of Russian Acad. of Sciences, 117810 Moscow, Russia
}

\begin{abstract}
Preliminary results are presented of the first (searching) stage of our observational program of the search and study of weak blue star-like objects in the two small $\gamma$-ray burst boxes GB 790418 and GB 790613. The basic search purposes are (1) the selection of star-like objects weaker than $23^{\mathrm{m}}$ inside these boxes, (2) the selection of the bluest ones in $B-V$ among these objects. The first result is the fact that there are no blue (with $B-V<0$ ) star-like objects brighter than $24^{\mathrm{m}}$ in both boxes. But in each of these GRB error boxes one blue object with smaller flux was selected for further study to elucidate their nature. In the smallest of the archival GRB error boxes (GB 790613) obtained by three satellite triangulation, the bluest star-like object with $V=24.56( \pm 0.16)$ and $B-V=-0.20( \pm 0.19)$ is near the center (at the distance $<10^{\prime \prime}$ ) of the GRB box. In the GB 790418 error box, the bluest star-like object with $B=24.40( \pm 0.20)$, $B-V=-0.40( \pm 0.40)$ is found at $\approx 50^{\prime \prime}$ from the center.
\end{abstract}

\section{Introduction}

If adhering to the idea of the Galactic origin of GRBs which proceeds from the concept of neutron-star type objects in the vicinity of the Sun, then such isolated, nearby objects should be found in the optical band as relatively blue, very faint $(V>23)$ ones with significant proper motions (Helfand et al. 1980). Of course, only the nearest objects can be seen at optical wavelengths. For example, in the "Local Disk models" (Hartmann 1994), these can be those up to $100 \mathrm{pc}$. A part of these nearest objects could appear as soft X-ray sources due to their thermal emission coming from the total surface, plus a polar cap accretion of the interstellar medium because of a strong magnetic field $\left(B \approx 5 \times 10^{12} \mathrm{G}\right)$.

Since the search for GRB counterparts can be connected with the search for very faint star-like objects in visible band, deep CCD photometry with big telescopes in the smallest GRB fields is urgently needed. Up to now, such deep searches could be performed only for a few well-localized GRB events. One example is the report by Vrba et al. (1994) who studied the GB 790613 and GB 790418 error boxes at the U.S. Naval Observatory $1.0 \mathrm{~m}$ telescope. But the search for very faint blue star-like candidates in fields investigated by small telescopes can become a basic task on a large telescope. 
In this respect, the observations at the $6 \mathrm{~m}$ telescope can be considered as a part of an observational program (together with deep X-ray observing, of course) for the direct verification of one of the galactic models. Though these are called in question now because of the $C G R O$ observations, they are not yet rejected completely. Even if we never find such weak and blue objects inside GRB boxes under consideration in a supposition of their relative nearness (50-100pc), then other models give even less hope to see anything in the optical band during the quiet state of the $\gamma$-ray bursters. Even in the "negative" case of no detections, we can say something about the upper limits of brightness of these objects for the Galactic GRB models.

Some preliminary results of the first ("searching") stage of two GRB fields can be reported. Here we only try to select the candidates for further study, or for the more precise $B V R$-photometry and proper motion study with the purpose of final elucidation of the nature of selected candidates.

\section{Observation}

CCD mosaic pointings for the two small GRB error boxes GB 790418 and GB 790613 were obtained at the $6 \mathrm{~m}$ telescope with a $580 \times 520$ pixel CCD in the primary focus ( $24 \mathrm{~m}$ ), providing $120 \times 80^{\prime \prime}$ field on the sky. The CCD chip has rectangular pixels of $24 \times 18 \mu \mathrm{m}$, resulting in an image scale of $0.205 \times 0$ ". $154 /$ pixel. Multiple observations (or CCD mosaic) with 600 and $400 \mathrm{sec}$ exposure times in the Cousins $B$ and $V$ filters were obtained.

Fig. 1 shows the error box for GB 790418 and the region actually covered by our CCD mosaic. The GRB error box was selected from the GRB catalogue (Atteia et al. 1987) and its configuration was taken from Golenetsky et al. (1986). The coordinates of the center of the error box are $\alpha(1950)=05^{\mathrm{h}} 51^{\mathrm{m}} 51^{\mathrm{s}} .84$; $\delta(1950)=-06^{\circ} 57^{\prime} 10^{\prime \prime} 8$. Our telescope+CCD combination required 7 pointings to cover almost the entire error box. Inside every frame its number is shown.

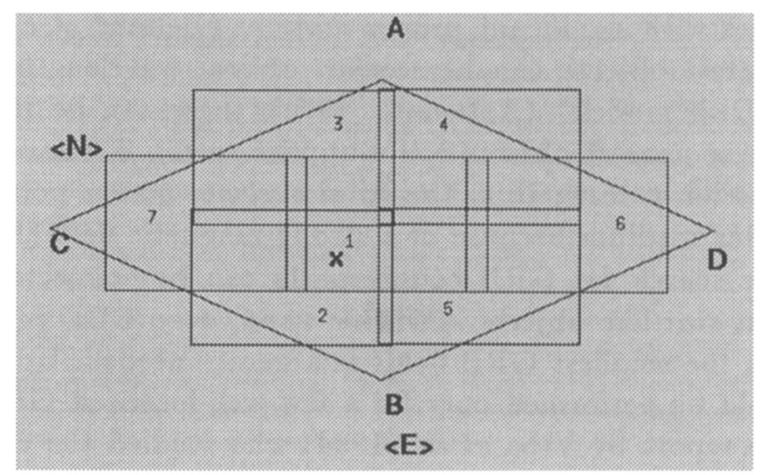

Fig. 1. CCD mosaic for the GB 790418 error box. 

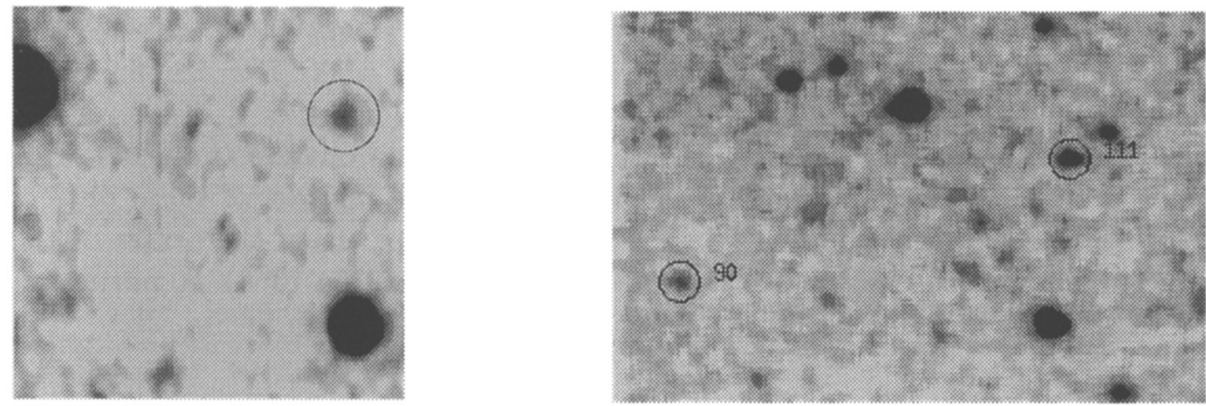

Fig. 2. Blow-ups of GB 790418 (left) and GB 790613 (right) CCD frames. Circle diameters are $6^{\prime \prime}$ and $4^{\prime \prime}$, respectively.

A portion of the central part of this CCD mosaic is shown in Fig. 2 (left, Gauss smoothed sum of all $B$ and $V$ frames). The seeing in the first night (February 6) was $2 . \prime 2-2.3$ in the central (1st and 2 nd) frames, and became a little better (about 1".9 FWHM) in the second night (Feb. 10). A relatively blue object, marked with a circle, is at a distance of approximately $50^{\prime \prime}$ from the GRB error box center. It seems to be star-like, when compared with the profiles of stars. Since the object is very close to the detection threshold its stellar nature cannot be asserted. to assert its star nature now. Its brightness and colour are $B=24.40 \pm 0.2, B-V=-0.40 \pm 0.40$. The object is apparently the bluest one in our CCD GRB field, in comparison with all 158 objects (including galaxies) of the field with $B<24.4$. The cross indicates theposition: $(\alpha(1950)=$ $\left.05^{\mathrm{h}} 51^{\mathrm{m}} 55^{\mathrm{s}} .28 ; \delta(1950)=-06^{\circ} 57^{\prime} 04^{\prime \prime} 3\right)$. In spite of a rather large colour error, it seems to deserve further efforts to elucidate its nature by measurements of its $B V R$ brightness and proper motion.

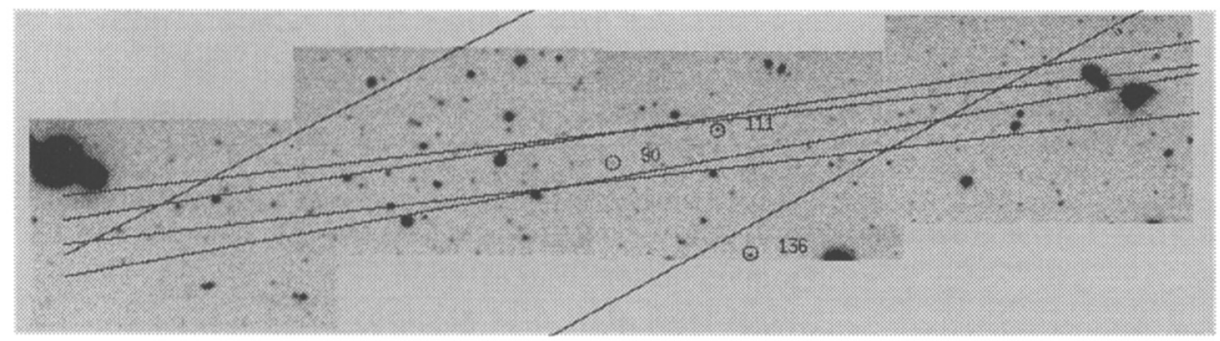

Fig. 3. The GB 790613 CCD field.

Our $B$ and $V$ CCD mosaic for the smallest GRB field (GB 790613) is shown in Fig. 3. The boundary of the symmetric GRB-error box is indicated according to the triangulation (see dicussion in Hack et al. 1994). Here also, each field is observed in two filters. The average seeing over the whole field did not exceed 
1"4. The observations were carried out on April 14, 1994. Three star-like and relatively blue objects are marked by circles: No. 90 with $B=24.36 \pm 0.10$, $B-V=-0.20 \pm 0.19$, No. 111 with $B=23.52 \pm 0.06, B-V=0.18 \pm 0.08$, and the brightest, evidently star-like object, No. 136 , being outside the GRB-error box $(B=23.14 \pm 0.03, B-V=-0.04 \pm 0.05)$. The faintest of these objects (No. 90) with a negative $B-V$ index lies within $\approx 10^{\prime \prime}$ from the center of the GRB-error box $\left(\alpha(1950)=14^{\mathrm{h}} 12^{\mathrm{m}} 12^{\mathrm{s}} 8, \delta(1950)=+78^{\circ} 54^{\prime} 13^{\prime \prime} 8\right)$. A portion of the central CCD frame with objects No. 90 and 111 is shown in Fig. $2 \mathrm{~b}$.

\section{Conclusion}

1. Inside both GRB error boxes, no blue star-like objects brighter than $B=24$ exist.

2. We selected the bluest candidates inside both GRB boxes for a more careful study (more precise photometry and proper motions) within our program on "The search for $\gamma$-ray bursters in a quiet state".

\section{References}

Atteia J.-L. et al., 1987, ApJS 64, 305

Golenetsky S.V. et al., 1986, preprint 1026, Ioffe PTI, Leningrad

Hartmann D.H., 1994, Proc. 2nd Huntsville GRB Workshop, AIP 307, p. 562

Hack F., et al., 1994, Proc. 2nd Huntsville GRB Workshop, AIP 307, p. 359

Helfand D.J., Chanan G.A., Novick R., 1980, Nature 289, 337

Vrba F.J., Hartmann D.H, Jennings M.C., 1994, Proc. 2nd Huntsville GRB Workshop, AIP 307, p. 443 